\title{
Influence of microstructural evolution on the magnetically group dominance in polycrystalline Y3Fe5O12 multi-samples
}

\begin{abstract}
In present work, the effect of changing microstructure on magnetic properties which evolves in parallel, in particular from amorphous-to-crystalline development, in yttrium iron garnet was investigated. 9 toroidal samples of polycrystalline yttrium iron garnets were prepared by using the mechanical alloying technique and sintered at low to high sintering temperature for microstructure-dependent-magnetic evolutions. A brief, yet revealing characterization of the samples were carried out by using an X-ray Diffraction, Field Emission Scanning Electron Microscopy, Impedance Material Analyzer, LCR-meter and, Picoammeter. It is believed that microstructural features such as amorphous phase, grain boundary, secondary phase and intergranular pores contribute significant additional magnetic anisotropy and demagnetizing fields, thus affecting the initial permeability accordingly. A scrutinizing observation of the permeability component results show that they tend to fall into three groups of magnetic permeability according to degree of magnetic behaviour dominance. The Curie temperature remained relatively stable and unaffected by the evolution, thus confirming its intrinsic character of being dependent only on the crystal structure and compositional stoichiometry. The increased electrical resistivity while the microstructure was evolving is believed to strongly indicate improved phase purity and compositional stoichiometry.
\end{abstract}

Keyword: Electrical properties; Magnetic materials; Magnetic properties; Microstructure 